\title{
ultramicroscopy
}

\section{Heating of TEM specimens during ion milling}

\author{
B. Viguier*, A. Mortensen \\ Laboratoire de Métallurgie Mécanique, Department of Materials, Swiss Federal Institute of Technology in Lausanne (EPFL), \\ CH 1015 Lausanne, Switzerland
}

Received 3 May 2000; received in revised form 1 September 2000

\begin{abstract}
Sample heating during preparation of electron-thin specimens for observation in transmission electron microscopy (TEM) can produce artefacts which invalidate observations. This is particularly true of two-phase materials such as metal matrix composites, for which sample cooling with liquid nitrogen cannot be used to preserve the substructure during milling. A series of experiments is conducted using an age-hardenable aluminium alloy which produces a trace of peak temperature attained by TEM specimens during ion milling. It is shown that peak temperatures of the order of $650 \mathrm{~K}$ can be attained using conventional milling parameters; the technique must therefore be used with caution with materials such as metal matrix composites. A simplified one-dimensional heat transfer analysis of the problem is conducted to show that the most critical parameter is heat transfer along the sample holder legs and across interfaces along the heat path. Temperature differentials within the TEM specimen are shown to be less significant, yet these alone are capable of reaching $60 \mathrm{~K}$ within a dimpled specimen under usual milling conditions. (C) 2001 Elsevier Science B.V. All rights reserved.
\end{abstract}

PACS: $61.16 . \mathrm{Bg} ; 81.050 . \mathrm{Ni} ; 61.80 . \mathrm{Jh}$

Keywords: Heating; Sample preparation; Ion milling; Metal matrix composite; Artefacts

\section{Introduction}

Transmission electron microscopy (TEM) is now well established as the principal method of high-magnification examination of materials microstructures; however, this technique is not free of potential problems. In particular, there is always

\footnotetext{
*Correspondence address. CIRIMAT/Laboratoire Interfaces et Matériaux, Ecole Nationale Supérieure de Chimie de Toulouse, INP, 118 route de Narbonne, 31077 Toulouse CEDEX 4, France. Tel.: + 33-5-62-88-56-64; fax: + 33-5-6288-56-63.

E-mail address: bviguier@ensct.fr (B. Viguier).
}

the risk that, either during examination using a microscope or during thinning of the sample to create the electron-transparent region of examination, artefacts may be introduced in the microstructure as a result of excessive sample heating.

In practice, this concern is often solved by experience-based knowledge that the sample never experiences, during both thinning and examination, temperatures sufficiently high to cause significant alterations or motion of those microstructural features that are to be examined. Otherwise, when heating may indeed pose a problem, nitrogen-cooled stages are used to keep the sample at all times sufficiently cold during 
thinning, so as to prevent thermally activated mechanisms from altering the microstructure that one desires to examine.

Unfortunately, with two-phase materials such as metal matrix composites, cold-stages often cannot be used to preserve the substructure of the material. Indeed, any significant temperature excursion, whether to higher or lower temperatures, is liable to generate internal stresses caused by thermal expansion misfit between the two phases forming these materials. These stresses, in turn, are susceptible to alter the dislocation structure within the more ductile metallic phases. To take a simple example, consider a material containing roughly equal volume fractions of metal and ceramic with a difference in coefficient of thermal expansion of $10^{-5} \mathrm{~K}^{-1}$ between the two phases. In this material, a temperature excursion of $100 \mathrm{~K}$ will generate internal misfit strains of the order of $0.1 \%$ : corresponding stresses will generally be sufficiently high to cause motion of dislocations in the metal matrix of such a material. Hence, if there is a tendency towards heating of the material during ion milling or during examination in TEM, cooling to liquid nitrogen temperatures does not provide an acceptable palliative.

For these reasons, only a few of the many TEM studies of metal matrix composites published to date can with confidence be deemed to be free of artefacts, either because jet polishing was used to thin the matrix (e.g., Refs. [1-5]) or because dislocations were decorated with matrix precipitates before thinning [6,7]. Ion milling, used in the vast majority of TEM examinations of metal matrix composites, is nonetheless a far more convenient technique than the two above techniques used to date to circumvent this heating problem: jet-polishing leaves the reinforcement unthinned and therefore electron-opaque, while precipitate pinning of dislocations introduces an additional and delicate step in the specimen preparation procedure and can furthermore only be practiced with a few metals. It would be of interest, therefore, to understand better what are the thermal consequences of ion thinning in the preparation of TEM samples. In turn, this would allow to know, with some certainty and in quantitative terms, when this technique can be used to examine dislocation structures of materials such as metal matrix composites.

The general problem of sample heating during preparation of electron-thin areas by ion milling has been addressed by several authors. Overall, experimental evidence available indicates that heating during ion milling under conditions (of beam intensity and angle) typical of metallographic thinning can indeed be significant [8-12]. Work to date unfortunately also shows significant scatter in the data obtained, the temperature increase reached by the specimen for equivalent thinning procedures varying from less than $100 \mathrm{~K}$ up to more than $400 \mathrm{~K}$. Moreover, we have not found a sufficiently quantitative treatment of the topic to indicate pathways for safe preparation of electron-thin areas in two-phase materials such as metal matrix composites. This formed the motivation of the present examination of sample heating during ion milling for TEM sample preparation.

The approach we have adopted is as follows. We prepare and examine ion-thinned samples of an age-hardenable aluminium alloy having a sufficiently clear "signature" of exposure to a temperature of the order of about $500 \mathrm{~K}$ and above. Using this material, by conducting a simple analysis of the heat transfer problem at hand and by varying the nature of the feet of the sample holder of an ion-milling apparatus, we have inferred principal governing thermal parameters (the energy flux flowing from the ion beam into the material, and the total interfacial thermal barrier to heat flow across the stage). We then use these quantities and the simple conduction heat transfer model to examine the applicability of ion milling for thinning of TEM samples free of artefacts resulting from excessive heating.

\section{Experimental procedures}

Samples of aluminium alloy 2017, of nominal composition given in Table 1, were cut in the form of disks ( $3 \mathrm{~mm}$ diameter, $1 \mathrm{~mm}$ thick) and then solutionized through a heat treatment in prevacuum at $773 \mathrm{~K}$ for $3 \mathrm{~h}$ followed by a water quench. The discs were then ground down to a thickness of $100 \mu \mathrm{m}$ using abrasive paper, finishing 
Table 1

Nominal composition of the 2017 aluminium alloy (wt \%)

\begin{tabular}{lllllllll}
\hline $\mathrm{Al}$ & $\mathrm{Si}$ & $\mathrm{Fe}$ & $\mathrm{Cu}$ & $\mathrm{Mn}$ & $\mathrm{Mg}$ & $\mathrm{Cr}$ & $\mathrm{Zn}$ & $\mathrm{Ti}+\mathrm{Zr}$ \\
\hline Bal. & 0.8 & 0.7 & 4.5 & 1.0 & 1.0 & 0.1 & 0.25 & 0.25 \\
\hline
\end{tabular}

with 1200 grid paper. This condition is defined in what follows as the reference state of the material. All subsequent experiments were conducted within one week of the solution heat treatment.

Ion beam exposure experiments and ion mill thinning were performed using two apparatuses: the DuoMill ${ }^{\mathrm{TM}}$ and the Precision Ion Polishing System (PIPS ${ }^{\mathrm{TM}}$ ) both produced by Gatan (Pleasanton, CA). In both systems, the gun stability during thinning was checked by recording the electric current displayed by the system as a function of time. These currents are quite different in the two systems. In the DuoMill, two currents are measured: the total gun current and that going through the specimen. In the PIPS, the current displayed is that collected by a Faraday cage located beyond the specimen; this current is provided only as a check for proper operation of ion guns and does not allow quantitative estimation of the energy delivered by the guns. Given the more interesting nature of current data displayed by the DuoMill, most of the experiments were conducted on this system. All polishing was conducted with argon ions, using two guns simultaneously. The gun current was set to $0.5 \mathrm{~mA}$ per gun on the DuoMill, while variable parameters were the gun voltage and the incidence angle $\theta$ (defined as the angle between the ion beam and the tangent to the specimen surface, i.e. reducing $\theta$ means moving to grazing incidences).

Specimen holders differed with the apparatus used. On the DuoMill system, the specimen is held using either (i) the standard three-legged holder designed for use without cooling (referred to hereafter as the RT holder) or alternatively (ii) a modified copper-legged holder designed for use with a nitrogen cold-finger. In both cases, the specimen to be thinned, a $3 \mathrm{~mm}$ diameter disc is clamped between two molybdenum plates.
The two holders differ by the material and the geometry of the legs. The RT holder has three legs made of steel $1.5 \mathrm{~mm}$ in diameter and $13 \mathrm{~mm}$ long. The copper legs designed for liquid nitrogen cooled thinning operation are usually somewhat longer than those made of steel in order to compensate for thermal contraction. For the present experiments, these legs were shortened so as to obtain proper sample positioning during thinning without nitrogen cooling. The copper holder used in the present study thus features three copper legs $13 \mathrm{~mm}$ long and of rectangular section, $2.2 \times 3 \mathrm{~mm}$.

In the PIPS system, experiments were conducted on both gripping and plate specimen holders provided by Gatan. For the plate holder, the specimens were pasted using an electrically and thermally conducting carbon-containing glue.

Electrolytic polishing was achieved with the double-jet technique, using a standard solution for aluminium thinning in a Tenupol ${ }^{\mathrm{TM}}$ (Struers, Denmark). TEM observations were performed using a CM20 Philips microscope operating at $200 \mathrm{kV}$ in the bright field two-beam mode using different diffraction vectors.

\section{Heat transfer analysis}

Calculations of specimen heating during the ion milling process are conducted by analysis of conductive heat transport along the specimen plus holder assembly, taking into account radiation thermal losses. Convection, on the other hand, is deemed insignificant since the ion milling process is conducted under high vacuum.

The specimen and holder assembly corresponding to the system used in the DuoMill is separated into three parts: the legs, the holder plates, and the specimen. The thermal conduction problem is simplified as follows, so as to render it one dimensional:

(i) the geometry of the assembly is rendered cylindrically symmetric. The three legs of the holder are, thus, assimilated to a cylindrical ring of identical length and cross-sectional area $\mathrm{A}$ as the three legs; and 
(ii) the temperature $T$ is supposed to be constant across the thickness of the specimen, and also across that of the holder, the plate support, and the legs (i.e., $T$ only depends on the lengthwise/radial position along the conduction column extending from the sample to the base plate).

The assumed overall geometry is depicted in Fig. 1. For the specimen, different configurations have been examined, comprising a flat specimen (of constant thickness) and specimens with dimpled profiles, such that the sample thickness $w$ is a function of radial position between $r=0$ and an outer radius $r=r_{d}$.

Boundary conditions are:

- a constant temperature $T_{0}=310 \mathrm{~K}^{1}$ in the base plate at the bottom of holder legs, taken to result from the relatively high thermal mass of the system from this point onwards,

- a total heat power $Q$ provided by the ion guns and supposed to be equally distributed under the ion beam of radius $r_{\mathrm{b}}$, so that the heat flux per second and unit area is constant under the beam $q=Q /\left(\pi r_{\mathrm{b}}^{2}\right)$ for $0 \leqslant r \leqslant r_{\mathrm{b}}$, and zero elsewhere.

The general governing equations for the temperature $T$ are further simplified by (i) focussing on steady-state conduction, and (ii) ignoring radiation losses from the TEM specimen itself, given its small surface area and the fact that these losses are masked by the overriding influence of the energy flux flowing into the material from the ion beam. The steady-state temperature distribution in the specimen is then governed by

$2 \pi r w(r) k_{\mathrm{s}} \frac{\partial T}{\partial r}=q \pi r^{2}$ for $r \leqslant r_{\mathrm{b}}$

and

$2 \pi r w(r) k_{\mathrm{s}} \frac{\partial T}{\partial r}=Q$ for $r_{\mathrm{b}}<r<r_{\mathrm{s}}$.

\footnotetext{
${ }^{1}$ In the DuoMill this temperature is due to the operation of vacuum diffusion pump in the cabinet close to the specimen chamber.
}

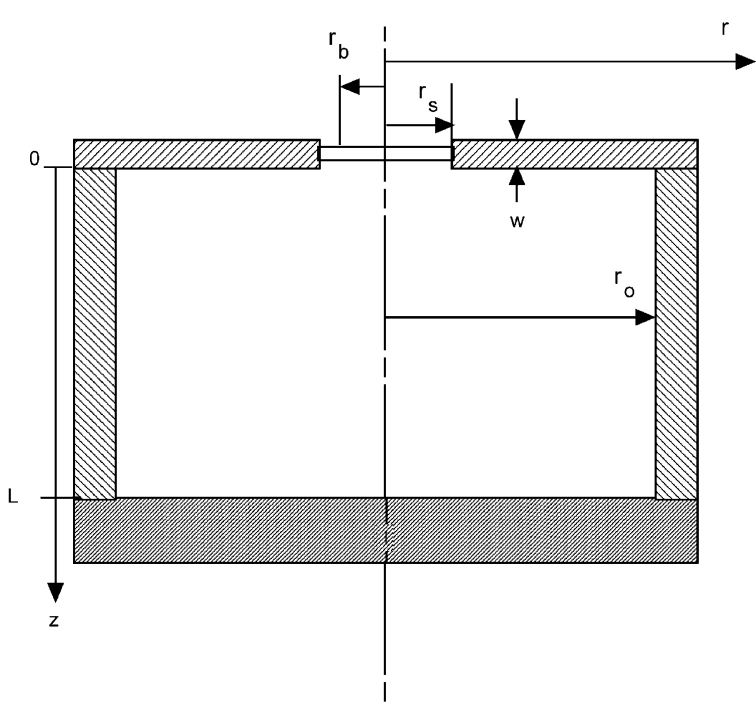

Fig. 1. Schematic of the specimen holder geometry assumed in the calculations.

For the holder plate and the legs, the steadystate heat transfer equations are, respectively,

$k_{\mathrm{h}} t\left(\frac{\partial^{2} T}{\partial r^{2}}+\frac{1}{r} \frac{\partial T}{\partial r}\right)=2 \sigma E_{\mathrm{h}}\left(T^{4}-T_{0}^{4}\right)$

and

$A k_{\ell} t \frac{\partial^{2} T}{\partial z^{2}}=P \sigma E_{\ell}\left(T^{4}-T_{0}^{4}\right)$.

In these equations $k$ refers to the thermal conductivity and $E$ to the emissivity with subscripts $\mathrm{s}, \mathrm{h}$ and $\ell$ referring to specimen, holder plate, and legs, respectively; $r_{\mathrm{s}}$ and $w(r)$ are the specimen radius and thickness, $t$ is the holder plate thickness, $A$ is the leg cross-sectional surface area, $P$ is the total leg periphery, $\sigma$ is the StefanBoltzmann constant, and $T_{0}$ is the ambient temperature in the apparatus.

Since the specimen is clamped in the holder plate which is simply attached with a bolt to each leg, we assume imperfect thermal contact at these locations. We account for this by assuming a finite interfacial heat transfer coefficient $h$ at these locations, taken to be identical at both interfaces. Boundary conditions of the problem are thus, for 
the specimen/holder interface:

$$
\begin{aligned}
h S_{1}\left(T_{\mathrm{h}, r_{\mathrm{s}}}-T_{\mathrm{s}, r_{\mathrm{s}}}\right) & =2 \pi r_{\mathrm{s}} w k_{\mathrm{s}}\left(\frac{\partial T_{\mathrm{s}}}{\partial r}\right)_{r=r_{\mathrm{s}}} \\
& =2 \pi r_{\mathrm{s}} t k_{\mathrm{h}}\left(\frac{\partial T_{\mathrm{h}}}{\partial r}\right)_{r=r_{\mathrm{s}}},
\end{aligned}
$$

where $S_{1}$ is the contact surface area between the specimen and the support plate of inner radius $r_{\mathrm{s}}$ see Fig. 1 (in calculations, $S_{1}$ was taken to correspond to the actual area of contact between sample and holder, taking into account the contact area on both sides of the sample). For the holder/ legs interface the boundary condition is

$$
\begin{aligned}
h A\left(T_{\ell, z=0}-T_{\mathrm{h}, r_{0}}\right) & =2 \pi r_{0} t k_{\mathrm{h}}\left(\frac{\partial T_{\mathrm{h}}}{\partial r}\right)_{r=r_{0}} \\
& =A k_{\ell}\left(\frac{\partial T_{\ell}}{\partial z}\right)_{z=0} .
\end{aligned}
$$

Integration of these equations is performed using Mathematica ${ }^{\mathrm{TM}}$ (Wolfram Research Inc., Champaign, Ill) on a personal computer, deducing $Q$ as a function of the sample peak temperature for each sample and holder configuration. Numerical values of material constants used in the calculation are reported in Table 2; the thermal emissivity coefficient was set to 0.5 for all materials.

\section{Experimental results}

The microstructure of the 2017 alloy after solution heat treatment and water quenching (Reference State) obtained from the solutionized disc solely by mechanical grinding and electrochemical thinning is presented in Fig. 2: helicoidal prismatic dislocation loops and some coarse intermetallic precipitates in a matrix free of any fine precipitation.

The correlation between precipitation state and temperature was established by heating discs for $3 \mathrm{~h}$ at each of the following temperatures: 323, 373, $453,513,573,623$ and $683 \mathrm{~K}$. For TEM observation the discs were electropolished using standard conditions.

After heat treatments at $453 \mathrm{~K}$ and below, no difference could be observed between the asquenched and heat treated states. For higher temperatures some precipitation could be observed. The corresponding microstructures are reported in Fig. 3. After aging at $513 \mathrm{~K}$, a very fine and homogeneous precipitation state is observed (Fig. 3a). For higher temperatures, coarsening of these precipitates is observed, the microstructure remaining homogeneous for heat treatment temperatures 573 and $623 \mathrm{~K}$, as illustrated in Figs. $3 \mathrm{~b}$ and c. After aging at $683 \mathrm{~K}$, very

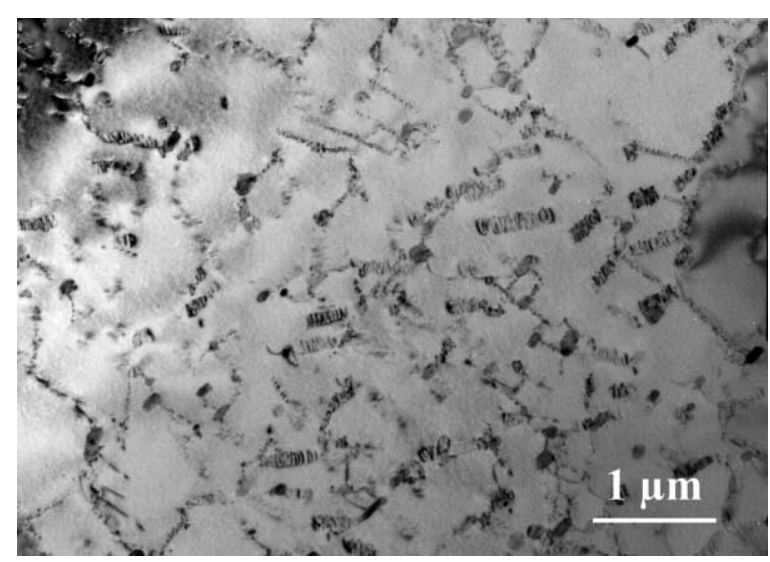

Fig. 2. Microstructure of 2017 alloy after solution heat treatment at $773 \mathrm{~K}$ for $3 \mathrm{~h}$. The specimen is thinned only by electropolishing.

Table 2

Values of materials constants used for the calculations [13]

\begin{tabular}{llccc}
\hline Part & Material & $k\left(\mathrm{~W} \mathrm{~m}^{-1} \mathrm{~K}^{-1}\right)$ & $\rho\left(\mathrm{kg} \mathrm{m}^{-3}\right)$ & $C_{\mathrm{p}}\left(\mathrm{J} \mathrm{kg}^{-1} \mathrm{~K}^{-1}\right)$ \\
\hline Specimen & Aluminium 2017 & 120 & - & - \\
Holder plate & Mo & 130 & 10.2 & 270 \\
Holder legs & Steel & 20 & 8.5 & 550 \\
Holder legs & $\mathrm{Cu}-\mathrm{Zn}$ & 150 & 8.75 & 380 \\
\hline
\end{tabular}



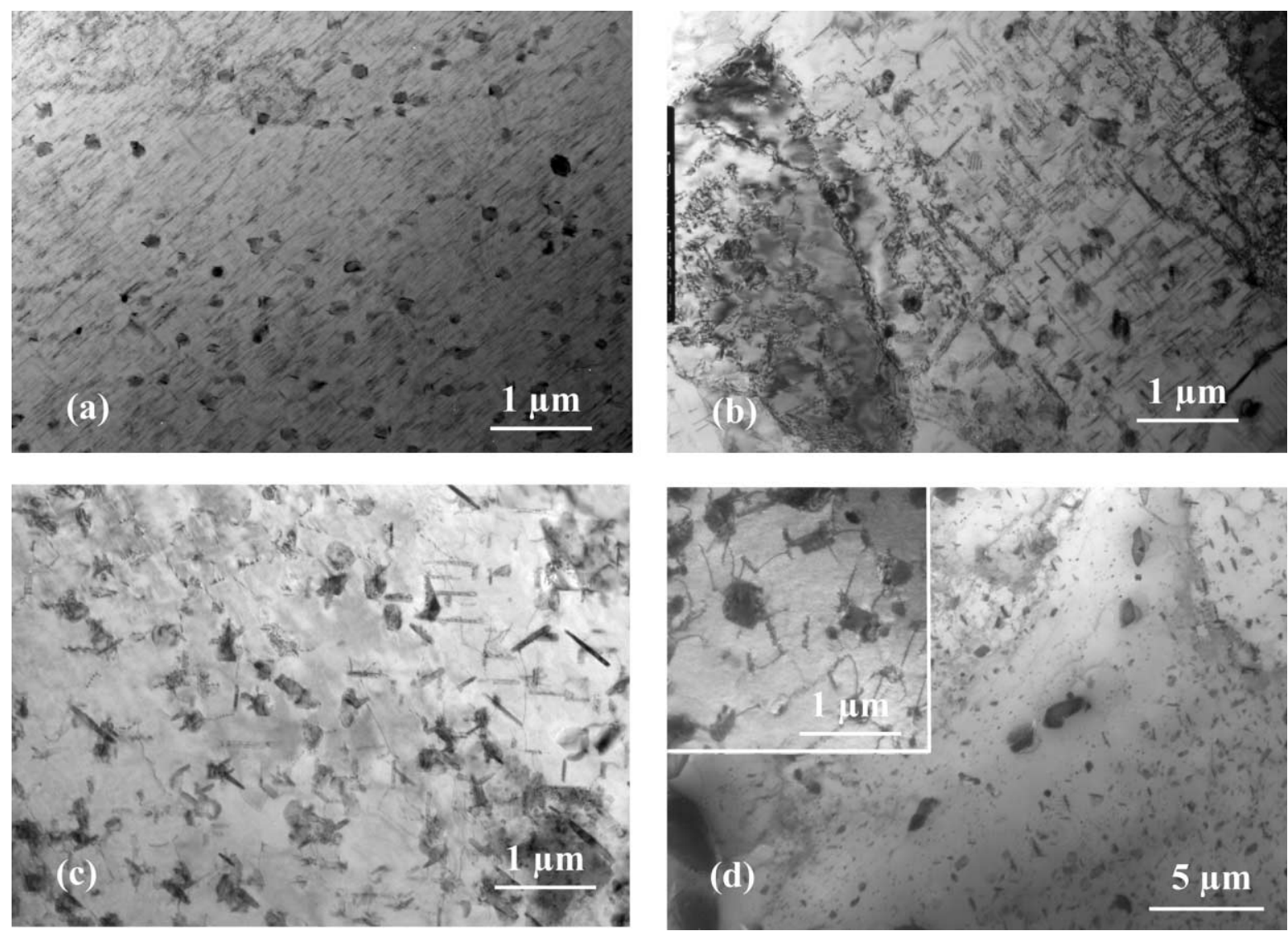

Fig. 3. Precipitation microstructure of 2017 alloy after solution treatment followed by isothermal heating for $3 \mathrm{~h}$ at different temperatures, the coarsening of precipitation serves as a temperature marker. (a) $513 \mathrm{~K}$, (b) $573 \mathrm{~K}$, (c) $623 \mathrm{~K}$ and (d) $683 \mathrm{~K}$. The specimen was thinned only by electropolishing.

coarse precipitates are also observed near the grain boundaries in Fig. 3d (note the different scaling in this image). Overall, the correlation between aging temperature and microstructure is relatively clear for temperatures between 453 and $683 \mathrm{~K}$.

Discs $(3 \mathrm{~mm}$ diameter and $100 \mu \mathrm{m}$ thick) that had been previously solution heat treated and quenched were subjected to ion milling in the DuoMill for $3 \mathrm{~h}$ under the following conditions: RT holder, two guns operating, total gun current $1 \mathrm{~mA}$, and incidence angle $\theta=15^{\circ}$. After this ion milling exposure, final polishing of the discs was performed electrolytically in order to obtain electron transparency. As seen in Fig. 4, the specimen submitted to the ion beam in the DuoMill features a precipitation microstructure that clearly does not compare with that of the same material thinned only electrolytically (Fig. 2). The precipitation structure observed in Fig. 4 corresponds roughly to that of the alloy aged at $683 \mathrm{~K}$ (Fig. 3d), as can be seen from the size and distribution of precipitates in the matrix and moreover by the presence of large precipitates near grain boundaries (see upper right corner of Fig. 4). This observation indicates that during ion milling with the above parameters, the temperature of this disk exceeded $673 \mathrm{~K}$. This experiment was then repeated: a total of five more specimens were treated and thinned under the same conditions. Three of the total of six discs thus produced exhibited the same microstructure as in Fig. 4; however, the three other specimens had a microstructure very 


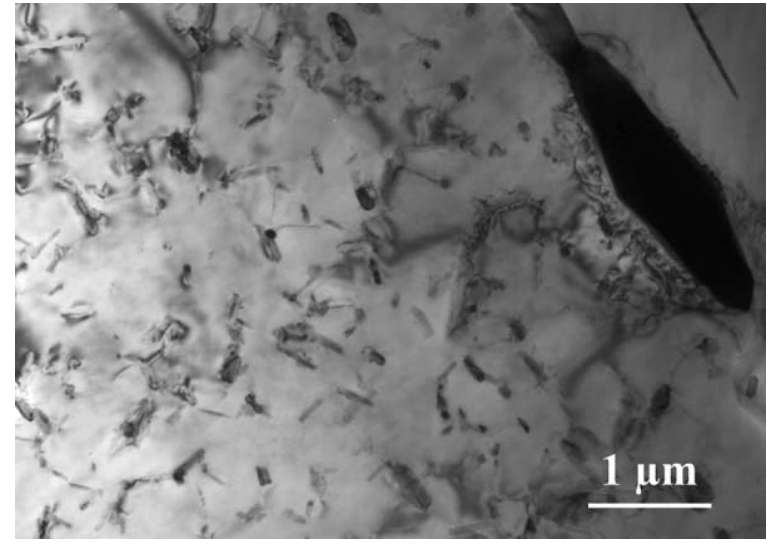

Fig. 4. Precipitation microstructure of 2017 alloy after solution heat treatment, the alloy was submitted to ion mill in the DuoMill for $3 \mathrm{~h}$, on a room temperature holder. Standard ion gun operating conditions are used : acceleration voltage $5 \mathrm{kV}$, guns current $1 \mathrm{~mA}$ and $\theta=15^{\circ}$.

similar to that corresponding to the as-quenched reference state shown in Fig. 2.

The procedure described above was repeated using different ion beam exposure conditions on the DuoMill. Four samples were milled under the same conditions as given above, save for the beam incidence angle $\theta$ which was altered from $15^{\circ}$ to $8^{\circ}$. Of these, the data again separated into two sets: two showed the initial precipitate-free microstructure, while two showed the structure given in Fig. 5, namely a fine and homogeneous precipitation of thin plates, quite similar to the precipitation state observed in Fig. 3b, which corresponds to aging at $573 \mathrm{~K}$. Decreasing the incidence angle $\theta$ from $15^{\circ}$ down to $8^{\circ}$ thus reduces the peak temperature reached by the specimen during ion milling from above $673 \mathrm{~K}$ to about $573 \mathrm{~K}$.

The same set of parameters was also used in the DuoMill with the copper specimen holder, featuring thicker legs made of copper instead of steel. In that case, it was observed by comparison with aged specimen microstructures that for $V=5 \mathrm{kV}$ and $\theta=15^{\circ}$, the disc reached a temperature of about $573 \mathrm{~K}$ for three out of four samples produced. With $V=5 \mathrm{kV}$ and $\theta=8^{\circ}$ no change in the microstructure could be detected during TEM observation of the three samples produced, indicating that the temperature remained below

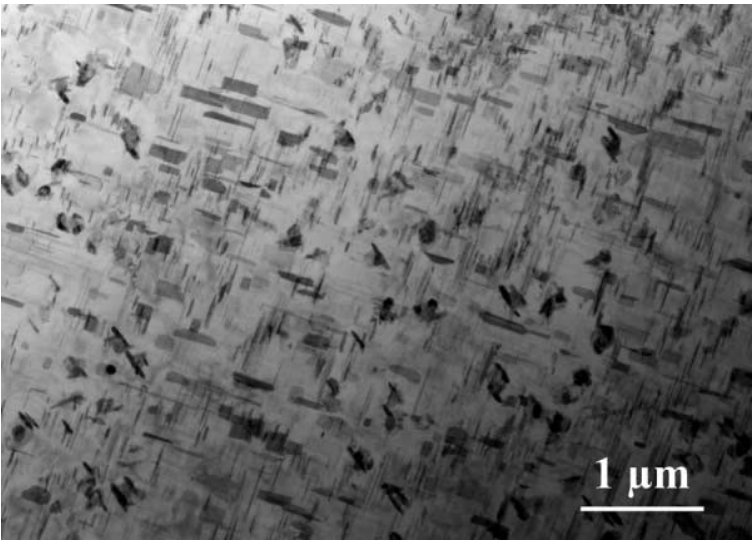

Fig. 5. Same as Fig. 4 but the incidence angle of the ion beam is reduced to $8^{\circ}$.

$500 \mathrm{~K}$ under these thinning conditions. The same conclusion was reached when ion milling was conducted at $V=3 \mathrm{kV}$ and $\theta=8^{\circ}$.

A similar exposure to the ion beam of flat $100 \mu \mathrm{m}$ thick discs was obtained in the PIPS using gripping holder system. These experiments were performed with a $5 \mathrm{kV}$ accelerating voltage and an incidence angle of $8^{\circ}$. The resulting microstructure is reported in Fig. 6 and consists of a very fine and homogenous precipitation of thin plates, similar to the microstructure observed in Fig. 3a. This experiment was repeated on six discs and such a microstructure was observed in three cases. The same experiment performed on two discs pasted on copper-based plates revealed no changes in the microstructure as compared to the reference state, indicating that the temperature did not exceed about $500 \mathrm{~K}$.

The results for all experiments are summarized in Table 3. An explanation for the separation into two batches of samples series apparently milled under identical conditions was sought for, by detailed examination of all data that could be obtained from the milling experiments. In particular, during the latter half of this study, detailed recordings of all milling parameters were conducted during the experiments. This led to the finding that cases for which the microstructure was left unchanged, denoting the absence of specimen heating during milling, corresponded to situations where the gun current was highly unstable in time, 
fluctuating constantly during the milling experiment.

In summary, thus, the following conclusions emerge from the experiments:

(i) temperatures exceeding $673 \mathrm{~K}$ can be reached within aluminium alloy specimens during ion milling conducted with quite usual parameter values $\left(5 \mathrm{kV}, i=1 \mathrm{~mA}, \theta=15^{\circ}\right)$,

(ii) the design and the material used for the specimen holder seem to be of prime importance in controlling the extent of specimen heating during milling, and

(iii) unsteady beam intensities lead to reduced specimen peak temperature as compared to stable gun operation.

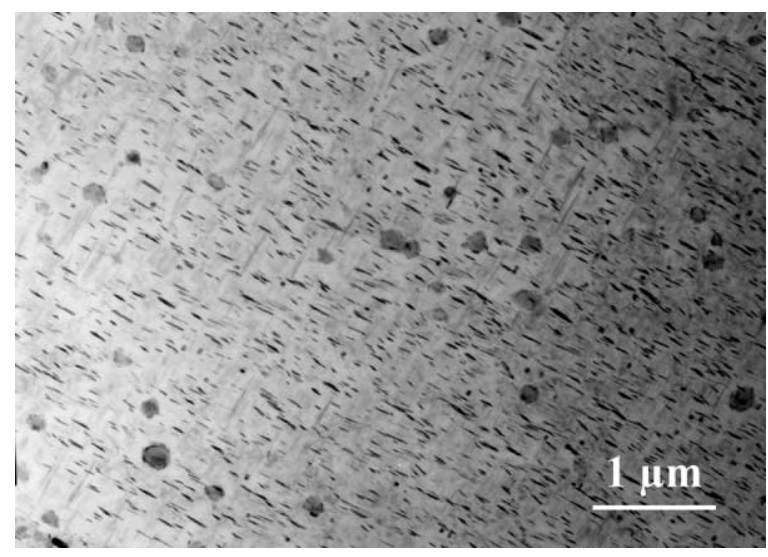

Fig. 6. Precipitation microstructure of 2017 alloy after solution heat treatment and ion mill polishing for $3 \mathrm{~h}$. The ion milling is realized on the PIPS apparatus, using two guns $\left(5 \mathrm{kV}, 8^{\circ}\right)$. The specimen is gripped on the DuoPost ${ }^{\mathrm{TM}}$ Gatan holder.

\section{Discussion}

The first conclusion from the several samples examined in this work is the binary nature of structures observed. For each condition potentially leading to sample heating, samples show the same high-temperature aged structure in about half the samples, and no alteration of the assolutionized structure for the other half. We attribute this, as mentioned, to fluctuating gun currents in experiments that lead to the production of non-heated samples. Samples which exhibit aged structures feature a similar aged structure for given milling conditions. We therefore conclude that these aged structures do, indeed, correspond to the peak temperature reached by the sample for steady-state milling under the conditions at hand.

If we compare samples milled in the DuoMill with an operating voltage of $5 \mathrm{kV}$ and an incidence angle of $15^{\circ}$, we see that the measured sample peak temperature decreases by $100 \mathrm{~K}$ when changing from steel to copper legs. Since differences between these two cases arise solely from the different thermal conductivity along the legs, their comparison can be used to determine parameters $Q$ and $h$.

The steady-state temperature profile was, thus, integrated for these two configurations and sets of parameters, seeking values for $Q$ and $h$ that satisfy the two peak temperature boundary conditions measured for the two cases. This led to the following values: $Q=1.6 \mathrm{~W}$ and $h=3700 \mathrm{~W} \mathrm{~m}^{-2} \mathrm{~s}^{-1} \mathrm{~K}^{-1}$. This value for $h$ is very reasonable, since it equals typical values for the heat transfer coefficient between metal and mould

Table 3

Summary of the maximum temperature reached by aluminium discs during ion milling under various conditions tested in this study

\begin{tabular}{lllrr}
\hline Apparatus & $\begin{array}{l}\text { Holder or specimen } \\
\text { fixing }\end{array}$ & $\begin{array}{l}\text { Operating voltage } \\
(\mathrm{kV})\end{array}$ & $\begin{array}{l}\text { Incidence angle } \\
\theta(\mathrm{deg})\end{array}$ & $\begin{array}{l}\text { Temperature }(\mathrm{K}) \\
\left(N_{\text {obs }} / N_{\text {total }}\right)^{\text {a }}\end{array}$ \\
\hline DuoMill & RT-steel-Mo & 5 & 15 & $673(3 / 6)$ \\
& & 5 & 8 & $573(2 / 4)$ \\
& Copper-Mo & 5 & 15 & $573(3 / 4)$ \\
& & 5 & 8 & $<500(3 / 3)$ \\
PIPS & Grip & 3 & 15 & $513(3 / 6)$ \\
& Pasted & 5 & 8 & $<500(2 / 2)$ \\
\hline
\end{tabular}

\footnotetext{
${ }^{\text {a }} N_{\text {obs }}$ is the number of discs for which the given temperature was observed, over the total number of discs $\left(N_{\text {tot }}\right)$ tested in the same conditions.
} 
in pressure die casting, quoted as being about $4000 \mathrm{~W} \mathrm{~m}^{-2} \mathrm{~s}^{-1} \mathrm{~K}^{-1}$ [14]. The calculated temperature profile corresponding to both holder types are given in Fig. 7.

Conclusions that emerge from the analysis are thus that:

(i) a significant interfacial resistance to heat transfer (i.e., a finite value of $h$ ) must be assumed to account for the high peak temperatures attained;

(ii) although there is a significant temperature difference across the specimen, the largest temperature rise occurs along the legs when these are made of poorly conducting material, and also at interfaces.

The interfacial heat transfer resistance accounts for a temperature rise of $200 \mathrm{~K}$ under the present conditions; its influence is thus significant. By comparing the peak temperatures reached by samples thinned with steel legs for different beam conditions (Table 3), we also conclude that, as the beam incidence angle is altered from $15^{\circ}$ to $8^{\circ}$, the total energy entering the specimen per unit time is decreased by about $30 \%$, to become $Q=1.12 \mathrm{~W}$. This observed decrease in $Q$ with decreasing incidence angle $\theta$ agrees with prior findings [12].

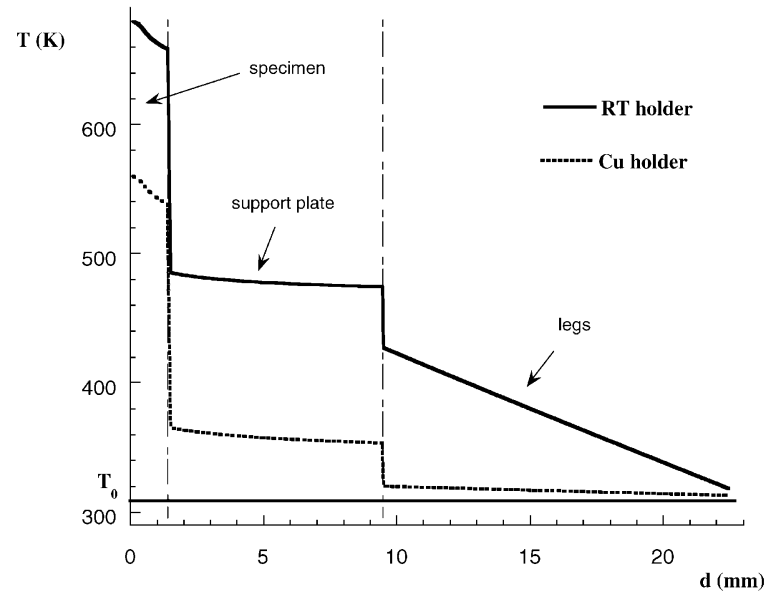

Fig. 7. Temperature profile along the conduction path (specimen, holder plate and holder legs) calculated for a $100 \mu \mathrm{m}$ flat disc maintained in both RT and copper holder (solid and dotted lines, respectively). Standard ion gun operating conditions are used: acceleration voltage $5 \mathrm{kV}$, guns current $1 \mathrm{~mA}$ and $\theta=15^{\circ}$.
The analysis was also used to explore to what extent the specimen shape may influence the temperature rise in the thinned section. Three sample geometries were compared: a flat cylindrical specimen and two dimpled specimen profiles depicted (with the beam) in Fig. 8(a), corresponding to linear and circular wedge profiles. The relative temperature increase in the specimen corresponding to a beam energy influx of $Q=1.6 \mathrm{~W}$ is plotted in Fig. 8(b). It is seen that, contrary to what is often postulated [12], the specimen thickness reduction during the milling process itself exerts only a limited influence on the peak sample temperature. Physically, this is because the beam energy input in the thinnest region is limited by the small surface area of this region. When changing from a flat specimen to a "triangular" dimple (this shape actually corresponds to the profile measured on flat specimens
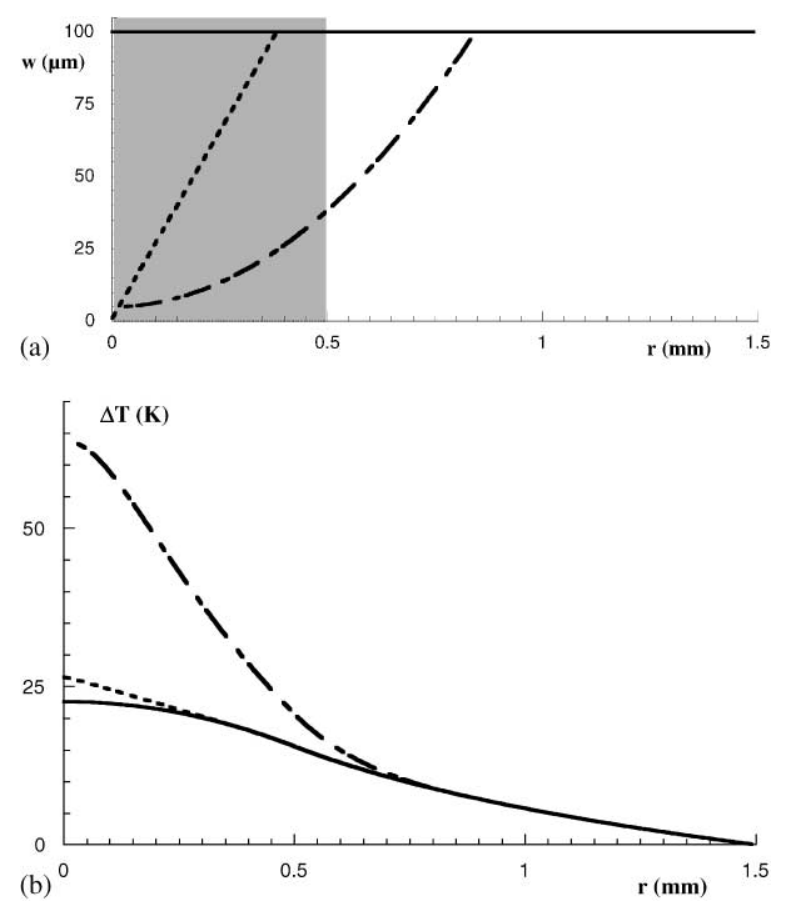

Fig. 8. Influence of sample geometry on the temperature increase during ion milling: (a) different thickness profiles (flat $(-)$, triangular dimple (---) and spherical dimple (-- -$)$ ), the grey area close to the specimen centre corresponds to the ion beam and (b) the corresponding relative temperature profile in the specimen, shown with the same lining. 
after ion-milling in the PIPS down to electron transparency) the temperature change in the specimen is only increased by $20 \%$. A more significant - but yet not drastic - increase in the maximum temperature at the specimen centre is obtained by simulation of a spherical dimple, corresponding to the specimen geometry resulting from mechanical dimpling, as usually performed in TEM specimen preparation. For an extreme case corresponding to a final specimen thickness of $5 \mu \mathrm{m}$ in the middle of the dimple, the temperature increase within the sample can reach $60 \mathrm{~K}$.

The present analysis is for steady-state conditions: without conducting the more involved heat transfer calculations that would be required to analyse unsteady situations (such as switch-on or switch-off of the ion mill, or the operation with an unstable ion gun). The system response to these transients can be assessed by estimation of the time $\Delta t$ for transfer of heat across the assembly, roughly given by

$\Delta t=\frac{\Delta L}{4 \alpha}$,

where $\alpha=k /\left(\rho C_{\mathrm{p}}\right)$ is the thermal diffusivity of the more heat-resistant material along the conduction column, and $\Delta L$ is the distance that must be travelled by the heat wave. Taking values for molybdenum, steel and brass, respectively, given in Table 2 with $\Delta L=1 \mathrm{~cm}$, we find $\Delta t \approx 0.5,60$ and $0.5 \mathrm{~s}$, respectively. As a consequence, the transient for temperature equilibration upon switch-on or switch-off of the apparatus will be of the order of a second with copper legs, and a minute with steel legs. Interestingly, the duration of this transient is consistent with daily operator experience as regards switching off of the ion mill. Indeed, when controlling a specimen during the ion milling process, since it takes at least $30 \mathrm{~s}$ after switching off the guns to access the sample (the time necessary to raise the specimen, break the vacuum and open the chamber), the operator will feel that the holder is either cold, or "only warm" (a frequent observation), while the maximum temperature reached by the sample during milling could have been as high as $673 \mathrm{~K}$. In other words, a lukewarm or cold holder upon retrieval does not indicate that there is no problem with regard to specimen heating during milling, even with relatively non-conductive steel-legged sample holders.

Under higher-frequency oscillatory operating conditions, featuring current oscillations of frequency greater than about $1 \mathrm{~Hz}$, the thermal inertia of the system will dampen oscillations, such that the sample peak temperature will be roughly that corresponding to steady state under the average beam energy in question. With the same peak energy but very frequent oscillations, the total energy input will be approximately halved in comparison with steady-state operation. This, in turn, will result in a significantly reduced sample heating, in turn explaining why, under unstable gun current conditions, sample temperatures apparently did not exceed $473 \mathrm{~K}$ for nominal operating parameter values which at steady state could heat the sample to $673 \mathrm{~K}$. Division of the data into two sets, Table 3, can thus be rationalized on these simple estimations coupled with the observation that lack of heating resulted from unstable current operation. Oscillatory current conditions could thus represent an interesting pathway for high-energy milling while preventing sample heat-up.

\section{Conclusion}

Conclusions from the present calculations and measurements are in accord with those of Kim and Carpenter [8]: peak sample temperatures of about $673 \mathrm{~K}$ can be reached during ion milling under usual conditions, and the most critical parameter is the aptitude of the sample holder legs to transfer the heat flowing from the beam into the sample.

A simplified one-dimensional steady-state heat analysis of the problem further shows that

(i) Interfacial heat transfer, in all likelihood across leg/plate interfaces, contributes significantly to specimen temperature rise during milling. Ensuring good heat transfer through the use of welded contacts and highly conductive materials can cut down the majority of the sample temperature rise during milling. 
(ii) The thinned sample geometry influences the peak temperature, particularly after mechanical dimpling; however, its effect is seemingly smaller than that of imperfect heat transfer along the sample holder. Still, within the sample area under the beam, this effect alone can generate a temperature rise of about $60 \mathrm{~K}$ in the electron-thin area.

More detailed analysis is, however, needed to firm up these conclusions since only a limited set of conditions was explored and simplifications were used in the analysis.

As regards the ion milling of materials such as metal matrix composites, for which cryogenic temperatures also generate artefacts, it must be concluded that:

(i) if peak temperatures near $373 \mathrm{~K}$ can be tolerated, ion milling can be conducted to produce electron-thin areas provided the sample holder assembly is constructed, and verified, for adequate heat transfer. To this end, a continuous pathway through highly conductive material all the way from the specimen to the heat sink must be ensured;

(ii) if temperature excursions of the order of $100 \mathrm{~K}$ are unacceptable, as is for example the case for examination of mobile dislocation structures in reinforced low to medium flow stress metals, ion milling must be controlled with extreme care, or avoided altogether.

\section{Acknowledgements}

This work was funded by core funding of the Laboratory for Mechanical Metallurgy at EPFL. Most of the experiments and all the observations were realized in the Interdepartmental Center for Electron Microscopy of EPFL (CIME). We wish to express our gratitude to Gaston Peter and Danièle Laub of CIME for their fruitful and experienced technical assistance.

\section{References}

[1] C.T. Kim, J.K. Lee, M.R. Plichta, Metall. Trans. A 21 (1990) 673

[2] J.A. Isaacs, A. Mortensen, Metall. Trans. A 23 (1992) 1207.

[3] A.J. Wilkinson, G. Gonzalez, D.J. Dingley, J. Microscopy 126 (1993) 255.

[4] C.Y. Barlow, N. Hansen, Acta Metall. Mater. 43 (1995) 3633.

[5] C.Y. Barlow, Y.L. Liu, Acta Mater. 46 (1998) 5807.

[6] P.B. Prangnell, W.M. Stobbs, in: N. Hansen, D. JuulJensen, T. Leffers, H. Lilholt, T. Lorentzen, A.S. Pedersen, O.B. Pedersen, B. Ralph (Eds.), 12th Ris $\varnothing$ International Symposium on Metallurgy and Materials Science, Metal Matrix Composites-Processing, Microstructure and Properties, Proceedings Conference, Roskilde, Denmark, 1991, Ris $\varnothing$ National Laboratory, Roskilde, Denmark, pp. 603610 .

[7] P.B. Prangnell, T. Downes, W.M. Stobbs, P.J. Withers, Acta Metall. Mater. 42 (1994) 3425.

[8] M.J. Kim, R.W. Carpenter, Ultramicroscopy 21 (1987) 327.

[9] D. Bahnk, R. Hull, in: R. Anderson (Ed.), Specimen Preparation for Transmission Electron Microscopy of Materials II - MRS Symposium Series Vol. 199, Proceedings Conference, San Francisco, CA, USA, 1990, The Materials Research Society, Pittsburgh, PA, USA, pp. 253-261.

[10] D. Bahnk, R. Hull, in: R. Anderson, B. Tracy, J. Bravman (Eds.), Specimen Preparation for Transmission Electron Microscopy of Materials III - MRS Symposium Series Vol. 254, Proceedings Conference, San Francisco, CA, USA, 1992, The Materials Research Society, Pittsburgh, PA, USA, pp. 249-256.

[11] K. Barmak, D.A. Rudman, S. Foner, J. Electron Microsc. Tech. 16 (1990) 249.

[12] D.J. Barber, Ultramicroscopy 52 (1993) 101.

[13] H.E. Boyer, T.L. Gall (Eds.), Metals Handbook, Desk Edition, American Society for Metals, Metals Park, OH, 1985.

[14] M.C. Flemings, Solidification Processing, McGraw-Hill, New York, 1974, p. 14. 